\title{
TWO GENERALIZATIONS OF JACOBI'S DERIVATIVE FORMULA
}

\author{
Samuel Grushevsky and Riccardo Salvati Manni
}

\begin{abstract}
In this paper we generalize Jacobi's derivative formula, considered as an identity for theta functions with characteristics and their derivatives, to higher genus/dimension. By applying the methods developed in our previous paper [GSM04], several generalizations to Siegel modular forms are obtained. These generalizations are identities satisfied by theta functions with characteristics and their derivatives at zero. Equating all the coefficients of the Fourier expansion of these relations to zero yields non-trivial combinatorial identities.
\end{abstract}

\section{Definitions and notations}

We denote by $\mathcal{H}_{g}$ the Siegel upper half-space - the space of symmetric complex $g \times g$ matrices with positive definite imaginary part. For $\varepsilon, \delta \in(\mathbb{Z} / 2 \mathbb{Z})^{g}$, thought of as vectors of zeros and ones, $\tau \in \mathcal{H}_{g}$ and $z \in \mathbb{C}^{g}$, the theta function with characteristic $[\varepsilon, \delta]$ is

$$
\theta\left[\begin{array}{l}
\varepsilon \\
\delta
\end{array}\right](\tau, z):=\sum_{m \in \mathbb{Z}^{g}} \exp \pi i\left[{ }^{t}\left(m+\frac{\varepsilon}{2}\right) \tau\left(m+\frac{\varepsilon}{2}\right)+2{ }^{t}\left(m+\frac{\varepsilon}{2}\right)\left(z+\frac{\delta}{2}\right)\right] .
$$

A characteristic $[\varepsilon, \delta]$ is called even or odd depending on whether it is even or odd as a function of $z$, which corresponds to the scalar product $\varepsilon \cdot \delta \in \mathbb{Z} / 2 \mathbb{Z}$ being zero or one, respectively. The number of even (resp. odd) theta characteristics is $2^{g-1}\left(2^{g}+1\right)$ (resp. $\left.2^{g-1}\left(2^{g}-1\right)\right)$. For $\varepsilon \in(\mathbb{Z} / 2 \mathbb{Z})^{g}$ the second order theta function with characteristic $\varepsilon$ is

$$
\Theta[\varepsilon](\tau, z):=\theta\left[\begin{array}{l}
\varepsilon \\
0
\end{array}\right](2 \tau, 2 z) .
$$

A theta constant is the evaluation at $z=0$ of a theta function. We drop the argument $z=0$ in the notations for theta constants. Obviously all odd theta constants vanish identically, and thus there are $2^{g-1}\left(2^{g}+1\right)$ non-trivial theta constants with characteristics, and $2^{g}$ theta constants of the second order.

A triplet of characteristics $\left[\varepsilon_{1}, \delta_{1}\right],\left[\varepsilon_{2}, \delta_{2}\right],\left[\varepsilon_{3}, \delta_{3}\right]$ is called azygetic if

$$
(-1)^{\varepsilon_{1} \cdot \delta_{1}+\varepsilon_{2} \cdot \delta_{2}+\varepsilon_{3} \cdot \delta_{3}+\left(\varepsilon_{1}+\varepsilon_{2}+\varepsilon_{3}\right) \cdot\left(\delta_{1}+\delta_{2}+\delta_{3}\right)}=-1 \text {. }
$$

Received by the editors December 28, 2003.

First author partially supported by NSF Mathematical Sciences Postdoctoral Research Fellowship. 
A sequence of $2 g+2$ characteristics $\left[\varepsilon_{1}, \delta_{1}\right], \ldots,\left[\varepsilon_{2 g+2}, \delta_{2 g+2}\right]$ is called a special fundamental system if the first $g$ characteristics are odd, the remaining are even and any triple of characteristics in it is azygetic.

In the genus 1 case one of the main identities for theta functions is Jacobi's derivative formula:

$$
\left.\frac{\mathrm{d}}{\mathrm{d} z} \theta\left[\begin{array}{l}
1 \\
1
\end{array}\right](\tau, z)\right|_{z=0}=-\pi \theta\left[\begin{array}{l}
0 \\
0
\end{array}\right](\tau) \theta\left[\begin{array}{l}
1 \\
0
\end{array}\right](\tau) \theta\left[\begin{array}{l}
0 \\
1
\end{array}\right](\tau)
$$

There is a long history of possible generalizations of this formula to higher genus. We consider $g$ odd characteristics $\left[\varepsilon_{1}, \delta_{1}\right], \ldots,\left[\varepsilon_{g}, \delta_{g}\right]$, and define their jacobian determinant to be

$$
\begin{aligned}
& D\left(\left[\varepsilon_{1}, \delta_{1}\right], \ldots\left[\varepsilon_{g}, \delta_{g}\right]\right)(\tau):= \\
& \quad \pi^{-g} \operatorname{grad} \theta\left[\begin{array}{l}
\varepsilon_{1} \\
\delta_{1}
\end{array}\right] \wedge \operatorname{grad} \theta\left[\begin{array}{l}
\varepsilon_{2} \\
\delta_{2}
\end{array}\right] \wedge \cdots \wedge \operatorname{grad} \theta\left[\begin{array}{l}
\varepsilon_{g} \\
\delta_{g}
\end{array}\right](\tau, 0) .
\end{aligned}
$$

Essentially the problem of generalizing Jacobi's derivative formula consists in expressing some linear combinations of jacobian determinants of $g$ distinct odd theta functions as polynomials or rational functions in theta constants. When $g=2$, such formulas are due to Rosenhain, Frobenius, Thomae, Fay, Igusa: we refer to [Th870], [Fr885], [Fa79], [Ig80] and [SM83] for exact statements and a precise description of the situation. We recall from these works that there is a precise conjectural formula, which has been proven for $g \leq 5$. Moreover, for $g \leq 3$ the equality

$$
D\left(\left[\varepsilon_{1}, \delta_{1}\right], \ldots,\left[\varepsilon_{g}, \delta_{g}\right]\right)(\tau)= \pm \theta\left[\begin{array}{l}
\varepsilon_{g+1} \\
\delta_{g+1}
\end{array}\right](\tau) \ldots \theta\left[\begin{array}{l}
\varepsilon_{2 g+2} \\
\delta_{2 g+2}
\end{array}\right](\tau)
$$

holds if and only if the $2 g+2$ characteristics appearing in it form a special fundamental system.

Differential equations for genus 2 theta constants have also been studied by Ohyama [Oh96] and Zudilin [Zu00]; Grant [Gr88] obtains a nice relation involving only one partial derivative.

Generalizations of Jacobi's derivative formula in another direction, to higher level theta constants in one variable, are derived and discussed in [FK01] generalizing these to the higher genus would also be very interesting.

A different generalization of Jacobi's derivative formula involves higher order derivatives of theta functions. For example it makes sense in genus 1 to ask for the expression of

$$
\begin{aligned}
& \operatorname{det}\left(\begin{array}{rc}
\theta\left[\begin{array}{l}
0 \\
0
\end{array}\right](\tau) & \theta\left[\begin{array}{l}
1 \\
0
\end{array}\right](\tau) \\
\left.\frac{\mathrm{d}^{2}}{\mathrm{~d}^{2} z} \theta\left[\begin{array}{l}
0 \\
0
\end{array}\right](\tau, z)\right|_{z=0} & \left.\frac{\mathrm{d}^{2}}{\mathrm{~d}^{2} z} \theta\left[\begin{array}{l}
1 \\
0
\end{array}\right](\tau, z)\right|_{z=0}
\end{array}\right) \\
& =4 \pi i \operatorname{det}\left(\begin{array}{rr}
\theta\left[\begin{array}{l}
0 \\
0
\end{array}\right](\tau) & \theta\left[\begin{array}{l}
1 \\
0
\end{array}\right](\tau) \\
\frac{\mathrm{d}}{\mathrm{d} \tau} \theta\left[\begin{array}{l}
0 \\
0
\end{array}\right](\tau) & \frac{\mathrm{d}}{\mathrm{d} \tau} \theta\left[\begin{array}{l}
1 \\
0
\end{array}\right](\tau)
\end{array}\right)
\end{aligned}
$$


as a polynomial in theta constants and first-order derivatives with respect to $z$ of theta functions, evaluated at $z=0$ (the two determinants are equal by the heat equation). We know in fact that

$$
\left.-\Theta[1](2 \tau)^{2} \frac{\mathrm{d}}{\mathrm{d} \tau}(\Theta[0](\tau)) / \Theta[1](\tau)\right)=\frac{i}{4 \pi}\left(\left.\frac{\mathrm{d}}{\mathrm{d} z} \theta\left[\begin{array}{l}
1 \\
1
\end{array}\right](\tau, z)\right|_{z=0}\right)^{2}
$$

or, equivalently,

$$
\operatorname{det}\left(\begin{array}{rr}
\Theta[0](\tau) & \Theta[1](\tau) \\
\frac{\mathrm{d}}{\mathrm{d} \tau} \Theta[0](\tau) & \frac{\mathrm{d}}{\mathrm{d} \tau} \Theta[1](\tau)
\end{array}\right)=\frac{i}{4 \pi}\left(\left.\frac{\mathrm{d}}{\mathrm{d} z} \theta\left[\begin{array}{l}
1 \\
1
\end{array}\right](\tau, z)\right|_{z=0}\right)^{2}
$$

To prove this, one can invoke a modular argument, saying that both sides are modular of the same weight, thus proportional, and the constant can be easily computed. Of course such a proof is not very revealing, and thus obtaining another proof would be desirable. As explained to us by H. Farkas, this identity can also be deduced from theorem 5.3 in chapter 2 of [FK01] by applying Jacobi's triple product identity, changing to the argument $\tau / 2$ and then verifying the resulting identity combinatorially for each coefficient of the Fourier series.

In this paper we shall generalize both the identities (5) and (6) to higher genus. It would be interesting to understand the combinatorial meaning of these generalizations similarly to the one-variable identities above or obtain alternative combinatorial proofs, but these questions lie beyond the scope of the current work.

The main tool will be a consequence of Riemann's addition theorem relating the first $z$-derivatives of odd theta functions with characteristics to the second $z$-derivatives of second order theta functions. This has also been the main tool in our paper [GSM04], where we showed that generically a principally polarized abelian variety is uniquely determined by the gradients of odd theta functions at $z=0$.

Remark 1. We note that the classical generalization of Jacobi's derivative formula can be given an interpretation in terms of theta series with harmonic polynomial coefficients. In fact monomials of degree $g+2$ in the theta constants are theta series relative to the quadratic form $4 \cdot 1_{g+2}$ and harmonic polynomial "1". The jacobian determinants, on the other hand, are theta series relative to the quadratic form $4 \cdot 1_{g}$ and harmonic polynomial "det", [Ig83].

This is the simplest pair of theta series with harmonic polynomial coefficients. Our generalizations can also be interpreted in this way. For example in genus one, while, as we wrote, in the first two cases the harmonic polynomials are 1 and $x$, in our case it is a polynomial in two variables: $x^{2}-y^{2}$.

As a further consequence of our formulas, we shall give a characterization of the locus of reducible principally polarized abelian variety in terms of vanishing of certain derivatives of odd theta functions. 


\section{The symplectic group action}

Let $\Gamma_{g}:=S p(2 g, \mathbb{Z})$ be the integral symplectic group; it acts on $\mathcal{H}_{g}$ by

$$
M \cdot \tau:=(A \tau+B)(C \tau+D)^{-1},
$$

where $M=\left(\begin{array}{cc}A & B \\ C & D\end{array}\right) \in \Gamma_{g}$. A period matrix $\tau$ is called reducible if there exists $M \in \Gamma_{g}$ such that

$$
M \cdot \tau=\left(\begin{array}{cc}
\tau_{1} & 0 \\
0 & \tau_{2}
\end{array}\right), \quad \tau_{i} \in \mathcal{H}_{g_{i}}, g_{1}+g_{2}=g ;
$$

otherwise we say that $\tau$ is irreducible.

We define the level subgroups of the symplectic group to be

$$
\begin{gathered}
\Gamma_{g}(n):=\left\{M=\left(\begin{array}{ll}
A & B \\
C & D
\end{array}\right) \in \Gamma_{g} \mid M \equiv\left(\begin{array}{ll}
1 & 0 \\
0 & 1
\end{array}\right) \bmod n\right\} \\
\Gamma_{g}(n, 2 n):=\left\{M \in \Gamma_{g}(n) \mid \operatorname{diag}\left(A^{t} B\right) \equiv \operatorname{diag}\left(C^{t} D\right) \equiv 0 \bmod 2 n\right\} .
\end{gathered}
$$

A function $F: \mathcal{H}_{g} \rightarrow \mathbb{C}$ is called a modular form of weight $k$ with respect to $\Gamma \subset \Gamma_{g}$ if

$$
F(M \cdot \tau)=\operatorname{det}(C \tau+D)^{k} F(\tau), \quad \forall M \in \Gamma, \forall \tau \in \mathcal{H}_{g}
$$

The theta functions transform under the action of $\Gamma_{g}$ as follows:

$$
\begin{aligned}
\theta\left[M\left(\begin{array}{l}
\varepsilon \\
\delta
\end{array}\right)\right]\left(M \cdot \tau,{ }^{t}(C \tau+D)^{-1} z\right) \\
\quad=\phi(\varepsilon, \delta, M, \tau, z) \operatorname{det}(C \tau+D)^{\frac{1}{2}} \theta\left[\begin{array}{l}
\varepsilon \\
\delta
\end{array}\right](\tau, z),
\end{aligned}
$$

where

$$
M\left(\begin{array}{l}
\varepsilon \\
\delta
\end{array}\right):=\left(\begin{array}{cc}
D & -C \\
-B & A
\end{array}\right)\left(\begin{array}{l}
\varepsilon \\
\delta
\end{array}\right)+\left(\begin{array}{l}
\operatorname{diag}\left(C^{t} D\right) \\
\operatorname{diag}\left(A^{t} B\right)
\end{array}\right)
$$

taken modulo 2 , and $\phi(\varepsilon, \delta, M, \tau, z)$ is some complicated explicit function. For more details, we refer to [Ig72] and [RF74].

Theta constants with characteristics are modular forms of weight $1 / 2$ with respect to $\Gamma_{g}(4,8)$. In this case $\phi(\varepsilon, \delta, M):=\phi(\varepsilon, \delta, M, \tau, 0)$ is an eighth root of unity that does not depend on $\tau$.

Differentiating the theta transformation law above with respect to some $z_{i}$ and then evaluating at $z=0$, we see that

$$
\begin{gathered}
\left.\frac{\partial}{\partial z_{i}} \theta\left[M\left(\begin{array}{l}
\varepsilon \\
\delta
\end{array}\right)\right](M \cdot \tau, z)\right|_{z=0} \\
=\left.\phi(\varepsilon, \delta, M) \operatorname{det}(C \tau+D)^{1 / 2} \sum_{j}(C \tau+D)_{i j} \frac{\partial}{\partial z_{j}} \theta\left[\begin{array}{l}
\varepsilon \\
\delta
\end{array}\right](\tau, z)\right|_{z=0} .
\end{gathered}
$$


Denoting by $\operatorname{grad} \theta\left[\begin{array}{l}\varepsilon \\ \delta\end{array}\right](\tau)$ the gradient of the theta function with respect to $z_{1}, z_{2}, \ldots, z_{g}$ at $z=0$, the above formula becomes

$$
\operatorname{grad} \theta\left[M\left(\begin{array}{l}
\varepsilon \\
\delta
\end{array}\right)\right](M \cdot \tau)=\phi(\varepsilon, \delta, M) \operatorname{det}(C \tau+D)^{\frac{1}{2}}(C \tau+D) \operatorname{grad} \theta\left[\begin{array}{l}
\varepsilon \\
\delta
\end{array}\right](\tau) .
$$

As a consequence, the jacobian determinant $D\left(\left[\varepsilon_{1}, \delta_{1}\right], \ldots\left[\varepsilon_{g}, \delta_{g}\right]\right)(\tau)$ is a modular form of weight $\frac{1}{2} g+1$ with respect to $\Gamma_{g}(4,8)$ (see [Ig80] and [SM83]).

\section{Some multilinear algebra}

For our purposes we need some results from linear algebra, which we recall and prove for the sake of completeness. We are grateful to C. De Concini, A. Maffei, D. Zagier and one of the referees for useful suggestions about these topics.

To any $A \in \operatorname{Mat}_{g \times g}(\mathbb{C})$ we associate the $(g-1) \times(g-1)$ matrix $\tilde{A}$ whose entries are the determinants of $2 \times 2$ minors of $A$ obtained taking the first line and the first column and letting the other row and column vary, i.e.

$$
\tilde{A}_{i j}:=\operatorname{det} A_{1 i}^{1 j}, \quad 2 \leq i, j \leq g .
$$

We observe that decomposing the matrix $A$ in blocks

$$
A=\left(\begin{array}{cc}
a_{11} & { }^{t} z \\
w & B
\end{array}\right)
$$

with $B$ a $(g-1) \times(g-1)$ matrix, and $z, w \in \mathbb{C}^{g-1}$, we have

$$
\tilde{A}_{i j}:=\operatorname{det}\left(a_{11} B-w^{t} z\right)
$$

With these notations we have

\section{Lemma 2.}

$$
a_{11}^{g-2} \operatorname{det} A=\operatorname{det} \tilde{A}
$$

Proof. It is trivial when $a_{11}=0$ and it is an immediate consequence of

$$
A=\left(\begin{array}{cc}
1 & 0 \\
w / a_{11} & \frac{1}{a_{11}} I
\end{array}\right)\left(\begin{array}{cc}
a_{11} & { }^{t} z^{\prime} \\
0 & \tilde{A}
\end{array}\right)
$$

when $a_{11} \neq 0$

We denote $N:=g(g+1) / 2$ and for any $v \in \mathbb{C}^{g}$ we denote by $v^{2}$ its symmetric tensor square. Then the following is true

Lemma 3. Let $v_{1}, \ldots, v_{N} \in \mathbb{C}^{g}$. Then

$$
\begin{gathered}
(N !) v_{1}^{2} \wedge v_{2}^{2} \wedge \cdots \wedge v_{N}^{2}=\sum_{s \in S_{N}} \operatorname{sign}(s)\left(v_{s(1)} \wedge v_{s(2)} \wedge \cdots \wedge v_{s(g)}\right) \\
\left(v_{s(1)} \wedge v_{s(g+1)} \wedge v_{s(g+2)} \wedge \cdots \wedge v_{s(2 g-1)}\right) \cdot\left(v_{s(2)} \wedge v_{s(g+1)} \wedge v_{s(2 g)} \wedge \cdots \wedge v_{s(3 g-3)}\right) \\
\left(v_{s(3)} \wedge v_{s(g+2)} \wedge v_{s(2 g)} \wedge \cdots \wedge v_{s(4 g-6)}\right) \ldots\left(v_{s(g)} \wedge v_{s(2 g-1)} \wedge v_{s(3 g-3)} \wedge \cdots \wedge v_{s(N)}\right)
\end{gathered}
$$


Proof. Since the LHS is $S L(g, \mathbb{C})$ invariant, it can be expressed as a polynomial in determinants of $g \times g$ minors of the $g \times N$ matrix with columns being $v$ 's. Moreover, this polynomial must be homogeneous of degree $g+1$ in these determinants, each $v_{i}$ has to appear in it exactly twice, and it has to be skewsymmetric in $v$ 's. For this reason first we sum over all possible permutations with the signs. We further observe that if the same two vectors appear in two different determinants, then the expression vanishes. Thus the expression has to be a sum of monomials each of degree $g+1$ in the determinants, such that each $v$ appears exactly twice, and no pair of $v$ 's appears twice in two different determinants. Thus the expression is forced to be exactly that of the statement, up to a multiplicative constant, which is easily computed.

\section{4. $\theta$ 's and $\Theta$ 's}

A special case of Riemann's bilinear addition theorem for theta functions (see [Ig72], [RF74],[Mu84]) is

$$
\Theta[\alpha](\tau, z) \Theta[\alpha+\varepsilon](\tau, 0)=\frac{1}{2^{g}} \sum_{\sigma \in(\mathbb{Z} / 2 \mathbb{Z})^{g}}(-1)^{\alpha \cdot \sigma} \theta\left[\begin{array}{l}
\varepsilon \\
\sigma
\end{array}\right](\tau, z) \theta\left[\begin{array}{l}
\varepsilon \\
\sigma
\end{array}\right](\tau, z)
$$

which is valid for all $\tau$ and $z$. Taking a sum of these with different signs, we get, for any $\delta \in(\mathbb{Z} / 2 \mathbb{Z})^{g}$

$$
\begin{gathered}
\sum_{\alpha \in(\mathbb{Z} / 2 \mathbb{Z})^{g}}(-1)^{\alpha \cdot \delta} \Theta[\alpha](\tau, z) \Theta[\alpha+\varepsilon](\tau, 0)= \\
\frac{1}{2^{g}} \sum_{\alpha, \sigma \in(\mathbb{Z} / 2 \mathbb{Z})^{g}}(-1)^{\alpha \cdot(\sigma+\delta)} \theta\left[\begin{array}{l}
\varepsilon \\
\sigma
\end{array}\right](\tau, z) \theta\left[\begin{array}{l}
\varepsilon \\
\sigma
\end{array}\right](\tau, z)=\theta\left[\begin{array}{l}
\varepsilon \\
\delta
\end{array}\right](\tau, z) \theta\left[\begin{array}{l}
\varepsilon \\
\delta
\end{array}\right](\tau, z) .
\end{gathered}
$$

We assume that the characteristic $[\varepsilon, \delta]$ is odd, differentiate this relation with respect to $z_{i}$ and $z_{j}$, and then evaluate at $z=0$. Denoting by $\mathbf{C}_{\varepsilon \delta}(\tau)$ the $g \times g$ symmetric matrix with entries

$$
\mathbf{C}_{\varepsilon \delta, i j}(\tau):=2 \partial_{z_{i}} \theta\left[\begin{array}{l}
\varepsilon \\
\delta
\end{array}\right](\tau, 0) \partial_{z_{j}} \theta\left[\begin{array}{l}
\varepsilon \\
\delta
\end{array}\right](\tau, 0),
$$

and by $\mathbf{A}_{\varepsilon \delta}(\tau)$ — the $g \times g$ symmetric matrix with entries

$$
\mathbf{A}_{\varepsilon \delta, i j}(\tau):=\left(\partial_{z_{i}} \partial_{z_{j}} \Theta[\delta](\tau, 0)\right) \Theta[\varepsilon](\tau, 0)-\left(\partial_{z_{i}} \partial_{z_{j}} \Theta[\varepsilon](\tau, 0)\right) \Theta[\delta](\tau, 0),
$$

we thus have (see [GSM04]) - notice that $\mathbf{C}_{\varepsilon \delta}=0$ if $[\varepsilon, \delta]$ is even

\section{Lemma 4.}

$$
\mathbf{C}_{\varepsilon \delta}(\tau)=\frac{1}{2} \sum_{\alpha \in(\mathbb{Z} / 2 \mathbb{Z})^{g}}(-1)^{\alpha \cdot \delta} \mathbf{A}_{\varepsilon+\alpha \alpha}(\tau)
$$

and the "inverse"

\section{Lemma 5.}

$$
\mathbf{A}_{\varepsilon+\alpha \alpha}(\tau)=\frac{1}{2^{g-1}} \sum_{\beta \in(\mathbb{Z} / 2 \mathbb{Z})^{g}}(-1)^{\alpha \cdot \beta} \mathbf{C}_{\varepsilon \beta}(\tau)
$$


We remark also that

$$
\mathbf{C}_{\varepsilon \delta}(\tau)=2 \operatorname{grad} \theta\left[\begin{array}{l}
\varepsilon \\
\delta
\end{array}\right](\tau){ }^{t} \operatorname{grad} \theta\left[\begin{array}{l}
\varepsilon \\
\delta
\end{array}\right](\tau) .
$$

\section{Generalized Jacobi's derivative formulas}

To generalize the first result of the introduction, we introduce the matrixvalued differential operator

Then we have

$$
\mathcal{D}:=\left(\begin{array}{cccc}
\frac{\partial}{\partial \tau_{11}} & \frac{1}{2} \frac{\partial}{\partial \tau_{12}} & \cdots & \frac{1}{2} \frac{\partial}{\partial \tau_{1 g}} \\
\frac{1}{2} \frac{\partial}{\partial \tau_{21}} & \frac{\partial}{\partial \tau_{22}} & \cdots & \frac{1}{2} \frac{\partial}{\partial \tau_{2 g}} \\
\ldots & \cdots & \cdots & \cdots \\
\frac{1}{2} \frac{\partial}{\partial \tau_{g 1}} & \cdots & \cdots & \frac{\partial}{\partial \tau_{g g}}
\end{array}\right) .
$$

Theorem 6 (First generalization). For any $\varepsilon \neq \delta$ the following holds:

$$
\begin{aligned}
& \mathrm{c} \Theta[\delta]^{2 g} \operatorname{det}(\mathcal{D}(\Theta[\varepsilon] / \Theta[\delta]) \\
& =\sum_{\left\{\alpha_{i_{1}}, \ldots, \alpha_{i_{g}} \mid\left[\varepsilon+\delta, \alpha_{i_{j}}\right] \text { odd }\right\}}(-1)^{\delta \cdot\left(\alpha_{i_{1}}+\cdots+\alpha_{i_{g}}\right)} D\left(\left[\varepsilon+\delta, \alpha_{i_{1}}\right], \ldots\left[\varepsilon+\delta, \alpha_{i_{g}}\right]\right)^{2}
\end{aligned}
$$

for some computable constant $\mathrm{c}$.

Proof. For any characteristics $\varepsilon, \delta$ we have by definition

$$
\Theta[\delta]^{2} \mathcal{D}(\Theta[\varepsilon] / \Theta[\delta])(\tau)=4 \pi i \mathbf{A}_{\varepsilon \delta}(\tau)
$$

Thus

$$
\Theta[\delta]^{2 g} \operatorname{det}(\mathcal{D}(\Theta[\varepsilon] / \Theta[\delta]))(\tau)=(4 \pi i)^{g} \operatorname{det}\left(\mathbf{A}_{\varepsilon \delta}\right)(\tau) .
$$

Now, using the result of lemma 2 , we get

$$
\begin{gathered}
\left.\operatorname{det} \mathbf{A}_{\varepsilon \delta}(\tau)=\operatorname{det}\left(\frac{1}{2^{g-1}} \sum_{\{\alpha \mid[\varepsilon+\delta, \alpha] \text { odd }\}}(-1)^{\delta \cdot \alpha} \mathbf{C}_{\varepsilon+\delta, \alpha}\right)(\tau)\right)= \\
\operatorname{det}\left(\frac{1}{2^{g-1}} \sum_{\{\alpha \mid[\varepsilon+\delta, \alpha] \text { odd }\}}(-1)^{\delta \cdot \alpha} \operatorname{grad} \theta\left[\begin{array}{c}
\varepsilon+\delta \\
\alpha
\end{array}\right](\tau)^{t} \operatorname{grad} \theta\left[\begin{array}{c}
\varepsilon+\delta \\
\alpha
\end{array}\right](\tau)\right) .
\end{gathered}
$$

When we expand this determinant, each summand will be of the type

$$
\begin{gathered}
\operatorname{sign}(\mu)\left((-1)^{\delta \cdot \alpha_{1}} \partial_{\mu(1)} \theta\left[\begin{array}{c}
\varepsilon+\delta \\
\alpha_{1}
\end{array}\right](\tau) \cdots(-1)^{\delta \cdot \alpha_{g}} \partial_{\mu(g)} \theta\left[\begin{array}{c}
\varepsilon+\delta \\
\alpha_{g}
\end{array}\right](\tau)\right) \\
\operatorname{sign}(\sigma)\left(\partial_{\sigma(1)} \theta\left[\begin{array}{c}
\varepsilon+\delta \\
\alpha_{1}
\end{array}\right](\tau) \cdots \partial_{\sigma(g)} \theta\left[\begin{array}{c}
\varepsilon+\delta \\
\alpha_{n}
\end{array}\right](\tau)\right)
\end{gathered}
$$

for some permutations $\sigma$ and $\mu$. Taking the sum of these for all possible permutations $\sigma$ and $\mu$ gives exactly the square of the jacobian determinant, so that we end up with

$$
\left(\frac{\pi^{2}}{2^{g-2}}\right)^{g} \sum_{\alpha_{i_{1}}, \ldots, \alpha_{i_{g}} \in(\mathbb{Z} / 2)^{g}}(-1)^{\delta \cdot\left(\alpha_{i_{1}}+\cdots+\alpha_{i_{g}}\right)} D\left(\left[\varepsilon+\delta, \alpha_{i_{1}}\right], \ldots\left[\varepsilon+\delta, \alpha_{i_{g}}\right]\right)^{2},
$$


proving the theorem.

At this point, we observe that our relations are not trivial. In fact each term appearing in the RHS is not identically zero, [SM83]. We remark that the set of characteristics appearing in the jacobian determinant above is syzygetic, while in all the other generalizations of Jacobi's derivative formula only azygetic sets appear, cf. [Ig80].

If we would like to have relations involving the derivatives of the second order theta constants with respect to $\tau_{i j}$, then since the matrix $\mathbf{C}_{\varepsilon \delta}(\tau)$ has rank 1 , we have the following

Proposition 7. If $g \geq 2$, then

$$
\operatorname{det}\left(\sum_{\alpha \in(\mathbb{Z} / 2 \mathbb{Z})^{g}}(-1)^{\alpha \cdot \delta} \mathbf{A}_{\varepsilon+\alpha \alpha}(\tau)\right)=0 .
$$

For the LHS of (13), we have the following non-vanishing:

Theorem 8. For all possible pairs $\varepsilon \neq \delta \in(\mathbb{Z} / 2 \mathbb{Z})^{g}$ the expression

$$
\Theta[\delta]^{2 g} \operatorname{det}(\mathcal{D}(\Theta[\varepsilon] / \Theta[\delta]))
$$

is not identically zero in $\tau$.

Proof. We shall prove a slightly more general result: that for any pair of distinct even characteristics $[\varepsilon, \alpha]$ and $[\delta, \beta]$ the expression

$$
\theta\left[\begin{array}{l}
\delta \\
\beta
\end{array}\right]^{2 g} \operatorname{det}\left(\mathcal{D}\left(\theta\left[\begin{array}{l}
\varepsilon \\
\alpha
\end{array}\right] / \theta\left[\begin{array}{l}
\delta \\
\beta
\end{array}\right]\right)\right)(\tau)
$$

is not identically zero.

Indeed, we know that the symplectic group $\Gamma_{g}$ acts doubly transitively on the set of even characteristics, and we have the following transformation formula

$$
\begin{gathered}
\mathcal{D}\left(\theta\left[M\left(\begin{array}{c}
\varepsilon \\
\alpha
\end{array}\right)\right] / \theta\left[M\left(\begin{array}{l}
\delta \\
\beta
\end{array}\right)\right]\right)(M \cdot \tau) \\
=\phi(\varepsilon, \alpha, \delta, \beta, M)(C \tau+D)^{t} \mathcal{D}\left(\theta\left[\begin{array}{l}
\varepsilon \\
\alpha
\end{array}\right] / \theta\left[\begin{array}{l}
\delta \\
\beta
\end{array}\right]\right)(\tau)(C \tau+D) .
\end{gathered}
$$

with $\phi(\varepsilon, \alpha, \delta, \beta, M)$ an eighth root of unity.

We learnt from [BZ03] that for some specific $[\varepsilon, 0]$ and $[\delta, 0]$ we have $\operatorname{det} \mathcal{D}\left(\theta\left[\begin{array}{l}\varepsilon \\ 0\end{array}\right] / \theta\left[\begin{array}{l}\delta \\ 0\end{array}\right]\right)$ not identically zero; since all such expressions are permuted by the symplectic group action, they are all not identically zero. 
For the second generalization of Jacobi's derivative formula, for any set of $N+1:=\frac{1}{2} g(g+1)+1$ characteristics $\varepsilon_{0}, \varepsilon_{1}, \ldots, \varepsilon_{N}$ we introduce the matrix

$$
M\left(\varepsilon_{0}, \varepsilon_{1}, \ldots, \varepsilon_{N}\right)(\tau):=\left(\begin{array}{ccc}
\Theta\left[\varepsilon_{0}\right] & \ldots & \Theta\left[\varepsilon_{N}\right] \\
\frac{\partial}{\partial \tau_{11}} \Theta\left[\varepsilon_{0}\right] & \cdots & \frac{\partial}{\partial \tau_{11}} \Theta\left[\varepsilon_{N}\right] \\
\frac{\partial}{\partial \tau_{12}} \Theta\left[\varepsilon_{0}\right] & \cdots & \frac{\partial}{\partial \tau_{12}} \Theta\left[\varepsilon_{N}\right] \\
\cdots & \cdots & \ldots \\
\frac{\partial}{\partial \tau_{g g}} \Theta\left[\varepsilon_{0}\right] & \cdots & \frac{\partial}{\partial \tau_{g g}} \Theta\left[\varepsilon_{N}\right]
\end{array}\right)
$$

It is a well-known fact (see [Sa83]) that $\operatorname{det} M\left(\varepsilon_{0}, \varepsilon_{1}, \ldots, \varepsilon_{N}\right)(\tau)$ is a modular form of weight $\frac{1}{4}(g+2)(g+3)$ relatively to $\Gamma_{g}(2,4)$. Moreover, it is not identically zero if and only if the theta constants

$$
\Theta\left[\varepsilon_{0}\right], \Theta\left[\varepsilon_{1}\right], \ldots, \Theta\left[\varepsilon_{N}\right]
$$

are algebraically independent. Indeed, if these theta constants are algebraically independent, they define a rational map from the modular variety to the projective space, the image of which is not contained in any closed subset. Thus the image is dense, and so there must exist an open subset in the modular variety where the map is locally invertible, and hence the rank of its differential is maximal. We set

$$
\delta_{k}:=\varepsilon_{0}+\varepsilon_{k}
$$

and let $\mathcal{C}_{\varepsilon \beta}(\tau)$ be the vector in $\mathbb{C}^{N}$ with entries $C_{\varepsilon \beta, i j}(\tau)$ (before we thought of $C$ as a matrix, but now we write down all the matrix elements in a single vector). Using the results of Lemmata 4, 2 and 1, we get the following

Proposition 9 (Second generalization). For some computable constant $c$

$$
=\sum_{\beta_{1}, \ldots, \beta_{N} \in(\mathbb{Z} / 2 \mathbb{Z})^{g}}^{c \Theta\left[\varepsilon_{0}\right]^{N-1} \operatorname{det} M\left(\varepsilon_{0}, \varepsilon_{1}, \ldots, \varepsilon_{N}\right)(\tau)}(-1)^{\varepsilon_{k} \beta_{k}} \operatorname{det}\left(\mathcal{C}_{\delta_{1} \beta_{1}} \wedge \mathcal{C}_{\delta_{2} \beta_{2}} \wedge \cdots \wedge \mathcal{C}_{\delta_{N} \beta_{N}}\right)
$$

By lemma 2 the RHS can be expressed as a homogeneous polynomial of degree $g+1$ in jacobian determinants.

Remark 10. Recalling the definition of theta functions, we can expand all of the above identities in Fourier series in $\tau$ or equivalently in power series in $q_{i j}:=\exp \tau_{i j}$. The coefficients of these expansions will then be some rather complicated but quite natural combinatorial quantities in several variables, and the equality of the RHS and LHS of any of the above would then yield a nontrivial multidimensional combinatorial identity, which it would be interesting to understand and prove combinatorially.

\section{An application in genus 2}

We will now work out in detail the situation in the case of genus 2 . Indeed let us write down (13) for $\varepsilon=[00]$ and $\delta=[10]$ :

$$
\Theta[10]^{4} \operatorname{det}\left(\mathcal{D}(\Theta[00] / \Theta[10])=c D([10,10],[10,11])^{2},\right.
$$


with $c$ a known constant.

Using lemma 4 to express the RHS in terms of theta constants of the second order and their derivatives, we get (we denote $\partial_{i j}:=\partial_{\tau_{i j}}$ to simplify notations)

$$
\begin{gathered}
\left(\Theta[00] \partial_{11} \Theta[10]-\Theta[10] \partial_{11} \Theta[00]\right)\left(\Theta[00] \partial_{22} \Theta[10]-\Theta[10] \partial_{22} \Theta[00]\right) \\
-\left(\Theta[00] \partial_{12} \Theta[10]-\Theta[10] \partial_{12} \Theta[00]\right)^{2} \\
+\left(\Theta[01] \partial_{11} \Theta[11]-\Theta[11] \partial_{11} \Theta[01]\right)\left(\Theta[01] \partial_{22} \Theta[11]-\Theta[11] \partial_{22} \Theta[01]\right) \\
-\left(\Theta[01] \partial_{12} \Theta[11]-\Theta[11] \partial_{12} \Theta[01]\right)^{2}=0
\end{gathered}
$$

Clearly we get the same equation if we chose $\varepsilon=[01]$ and $\delta=[11]$. Thus in all we get three different equations.

In [BZ03] it is shown that in genus 2 there are $2 \cdot 2^{2}+2=10$ algebraically independent quantities among 4 theta constants of the second order and their $4 \cdot 3=12$ derivatives. Thus there are 6 non-trivial algebraic relations among theta constants and their first-order derivatives. So far we have obtained three such equations, and three more can be obtained by writing down formula (14).

In [BZ03] some other 6 independent relations are given. We shall prove that they are all consequences of (13) and (3).

Indeed, for genus two formula (3) reads

$$
D([10,10],[10,11])^{2}=\left(\theta\left[\begin{array}{l}
11 \\
00
\end{array}\right] \theta\left[\begin{array}{l}
11 \\
11
\end{array}\right] \theta\left[\begin{array}{l}
01 \\
00
\end{array}\right] \theta\left[\begin{array}{l}
01 \\
10
\end{array}\right]\right)^{2} .
$$

Applying (9) to rewrite the RHS in terms of theta constants of the second order, we finally see that

$$
\Theta[10]^{4} \operatorname{det}(\mathcal{D}(\Theta[00] / \Theta[10])
$$

is a polynomial in the theta constants of the second order. This equation is up to a rational function equal to one of the equations in [BZ03]. From the other choices of characteristics $\varepsilon$ and $\delta$ we get the other 5 equations. We observe that these 5 equations can also be obtained from the first one by the action of $\Gamma_{2}$. In this spirit we remark that in genus 1 , we have four variables and the relation is an immediate consequence of (1) and (6). These relations can also be obtained considering the determinant of (14).

This method allows us to give a conjectural description of the situation in the genus 3 case. By the results of [BZ03] we know that among 56 variables (all $\Theta[\varepsilon]$ and their derivatives $\partial_{i j} \Theta[\varepsilon]$ ) there are 21 algebraically independent ones. Thus there are 35 algebraic relations. We know that there is a unique polynomial relation among the $\Theta[\varepsilon]$, of degree 16, cf. [vGvG86]; let us denote it by $R(\tau)$. Thus we have

$$
R(\tau)=0, \quad \partial_{i j} R(\tau)=0
$$

and other 28 relations obtained by applying (14), since in genus 3 there are exactly 28 odd characteristics. So in total we have 35 relations that we conjecture to be algebraically independent. 


\section{Characterization of the reducible locus}

We finish the paper by giving a characterization of the locus of reducible abelian varieties. Different characterizations of the reducible locus are known: in [EL97] it is characterized in terms of the dimension of the singular locus of the theta divisor, in [Sa83] — in terms of the non-maximality of the rank of matrix $P(\tau)$ with rows $\left(\Theta[\varepsilon], \frac{\partial}{\partial \tau_{i j}} \Theta[\varepsilon]\right)$, and columns corresponding to all $\varepsilon \in(\mathbb{Z} / 2 \mathbb{Z})^{g}$, in [SM94] — in terms of the vanishing of certain theta constants. Here we use the vanishing of certain first derivatives of theta functions evaluated at zero.

Proposition 11. A ppav with a period matrix $\tau$ is reducible if and only if there exist some $M \in \Gamma_{g}$ and some $k<g$ such that if we write any odd characteristic $[\varepsilon, \delta]$ as $\left[\varepsilon_{1} \varepsilon_{2}, \delta_{1} \delta_{2}\right]$, where $\left[\varepsilon_{1}, \delta_{1}\right]$ is a $k$-dimensional characteristic, and $\left[\varepsilon_{2}, \delta_{2}\right]$ is $(g-k)$-dimensional, then

$$
\left.\partial_{z_{i}} \theta\left[\begin{array}{l}
\varepsilon \\
\delta
\end{array}\right](M \cdot \tau, z)\right|_{z=0}=0
$$

for all $i \leq k$ for $\left[\varepsilon_{1}, \delta_{1}\right]$ even, and for all $i>k$ for $\left[\varepsilon_{1}, \delta_{1}\right]$ odd.

Proof. Suppose the period matrix $M \cdot \tau$ splits as $M \cdot \tau=\left(\begin{array}{cc}\tau_{1} & 0 \\ 0 & \tau_{2}\end{array}\right)$, with $\tau_{1} \in \mathcal{H}_{k}$ and $\tau_{2} \in \mathcal{H}_{g-k}$, so that the theta functions with characteristics factor as follows:

$$
\theta\left[\begin{array}{l}
\varepsilon \\
\delta
\end{array}\right](M \cdot \tau, z)=\theta\left[\begin{array}{l}
\varepsilon_{1} \\
\delta_{1}
\end{array}\right]\left(\tau_{1}, z_{1}\right) \cdot \theta\left[\begin{array}{l}
\varepsilon_{2} \\
\delta_{2}
\end{array}\right]\left(\tau_{2}, z_{2}\right) .
$$

The vanishing of the derivatives in question is immediate for $M \cdot \tau$ by differentiating and evaluating at $z=0$; thus the "only if" part is proven. For the "if" part, assume the vanishing of derivatives as stated. Then according to (11) we have $\mathbf{C}_{\varepsilon \delta, i j}=0$ and consequently $\mathbf{A}_{\varepsilon \delta, i j}=0$ for $1 \leq i \leq k<j \leq g$ and all odd $[\varepsilon, \delta]$. Thus the matrix $P(\tau)$ does not have maximal rank, and thus, by the results of [Sa83], it corresponds to a reducible abelian variety.

\section{References}

[BZ03] D. Bertrand and W. Zudilin, On the transcendence degree of the differential field generated by Siegel modular forms, J. Reine Angew. Math. 554 (2003), 47-68.

[EL97] L. Ein and R. Lazarsfeld, Singularities of theta divisors and the birational geometry of irregular varieties. J. Amer. Math. Soc. 10 (1997), no. 1, 243-258.

[Fa79] J. Fay On the Riemann-Jacobi formula. Nachr. Akad. Wiss. Gottingen Math.-Phys. Kl. II 1979, no. 5, 61-73.

[FK01] H. Farkas and I. Kra, Theta constants, Riemann surfaces and the modular group. An introduction with applications to uniformization theorems, partition identities and combinatorial number theory. Graduate Studies in Mathematics, 37. AMS, Providence, 2001.

[Fr885] G. Frobenius, Über die constanten Factoren der Thetareihen, J. Reine Angew. Math. 98 (1885) 244-265.

[vGvG86] B. van Geemen and G. van der Geer, Kummer varieties and the moduli spaces of abelian varieties, Amer. J. Math. 108 (1986) 615-641.

[Gr88] D. Grant, A generalization of Jacobi's derivative formula to dimension two, J. Reine Angew. Math. 392 (1988) 125-136. 
[GSM04] Grushevsky, S., Salvati Manni, R. Gradients of odd theta functions, J. Reine Angew. Math. 573 (2004) 43-59

[Ig72] J.-I. Igusa, Theta functions. Die Grundlehren der mathematischen Wissenschaften, Band 194. Springer-Verlag, New York-Heidelberg, 1972.

[Ig80] On Jacobi's derivative formula and its generalizations. Amer. J. Math. 102 (1980) 409-446.

[Ig83] Multiplicity one theorem and problems related to Jacobi's formula. Amer. J. Math. 105 (1983) 157-187.

[Mu84] D. Mumford, Tata lectures on Theta II. Progress in Math. vol. 43, Birkhäuser, Boston, 1984.

[Oh96] Y. Ohyama, Non-linear equations on theta constants of genus two. Preprint of Osaka University 1996.

[Pr82] C. Procesi, A Primer of Invariant Theory. Brandeis Lecture Notes 1 (1982).

[RF74] H. Rauch and H. Farkas, Theta functions with applications to Riemann surfaces. The Williams-Wilkins Co., Baltimore, 1974.

[SM83] R. Salvati Manni, On the nonidentically zero Nullwerte of Jacobians of theta functions with odd characteristics. Adv. in Math. 47 (1983), no. 1, 88-104.

[SM94] _ Modular varieties with level 2 theta structure. Amer. J. Math. 116 (1994), no. 6, 1489-1511.

[Sa83] R. Sasaki, Modular forms vanishing at the reducible points of the Siegel upper-half space, J. Reine Angew. Math. 345 (1983) 111-121.

[Th870] J. Thomae, Beitrag zur Bestimmung von $\theta(0,0, \ldots, 0)$ durch die Klassenmoduln algebraischer Funktionen, J. Reine Angew. Math. 71 (1870) 201-222.

[Zu00] W. Zudilin, Theta constants and differential equations Mat. Sb. 191 (2000) 77-122 (in Russian), translation in Russian Acad. Sci. Sb. Math.

Mathematics Department, Princeton University, Fine Hall, Washington Road, PRINCETON, NJ 08544

E-mail address: sam@math.princeton.edu

Dipartimento di Matematica, Università "La Sapienza", Piazzale A. Moro 2, Roma, I 00185, ITALY

E-mail address: salvati@mat.uniroma1.it 